\title{
Kikuchi-Fujimoto Disease Associated with Sjögren's Syndrome: A Case Report
}

\author{
Abdul-Wahab Al-Allaf, Yousef M. Yahia \\ Rheumatology Department, Hamad Medical Corporation, Doha, Qatar
}

How to cite this article: Al-Allaf AW, Yahia YM. Kikuchi-Fujimoto disease associated with Sjörgen syndrome: a case report. EJCRIM 2018;5: doi:10.12890/2018_000856.

Conflicts of Interests: The Authors declare that there are no competing interests.

This article is licensed under a Commons Attribution Non-Commercial 4.0 License

\section{ABSTRACT}

Kikuchi-Fujimoto disease (KFD) or Kikuchi histiocytic necrotizing lymphadenitis, is an extremely rare, benign and self-limiting disease which can be confused with lymphoma and sometime with infections such as tuberculosis. It has also been infrequently reported in association with autoimmune diseases. KFD shares sex and age predisposition as well as histological features with systemic lupus erythematosus. The co-existence of KFD with primary Sjögren's syndrome has only been rarely reported. Here we describe the case of a 33-year-old woman who was diagnosed with KFD and also found to have primary Sjögren's syndrome.

\section{LEARNING POINTS}

- To understand the relationship between Kikuchi-Fujimoto disease (KFD) and Sjögren's syndrome.

- KFD shares some characteristics with autoimmune diseases.

- It is important to maintain a high index of suspicion of Sjögren's syndrome and diffuse lymphadenopathy in patients with KFD.

\section{KEYWORDS}

Kikuchi-Fujimoto disease, Sjögren's syndrome, necrotizing histiocytic lymphadenitis

\section{CASE DESCRIPTION}

A 33-year-old Indian woman had a 10-year history of hyperprolactinaemia treated with cabergoline. She presented with a painless right inguinal lump of 2 months' duration, which was increasing in size but not tender. She felt generally well and reported no fever, night sweats, weight loss or any changes in appetite. Examination was unremarkable apart from a short history of dry eye. The patient denied recent travel or a history of tuberculosis. Ultrasound of the abdomen and pelvis revealed multiple enlarged necrotic-appearing retroperitoneal and right pelvic lymph nodes.

A CT of the chest and abdomen confirmed these findings. A biopsy of the right groin mass revealed multiple granulomas composed of epithelioid histiocytes and scattered giant cells with central areas of necrosis (Fig. 1). ZN staining was negative for acid-fast bacilli, Grocott staining was negative for fungi and cytology was also negative. The provisional diagnosis was Kikuchi-Fujimoto disease (KFD). However, in view of the patient's history of dry eye and lymphadenopathy, immunology testing was conducted and was positive for ANA speckled type, anti-RO and anti-LA with a high lgG of $2140 \mathrm{mg} / \mathrm{dl}$ and a high ESR of 47 . These findings supported a clinical and biochemical diagnosis of primary Sjögren's syndrome. Clinical and laboratory investigations did not suggest any other types of connective tissue disease. The renal profile, liver profile, ferritin, C3 and C4 were all normal. The patient was negative for anti-double strand DNA, ANCA and RNP. 

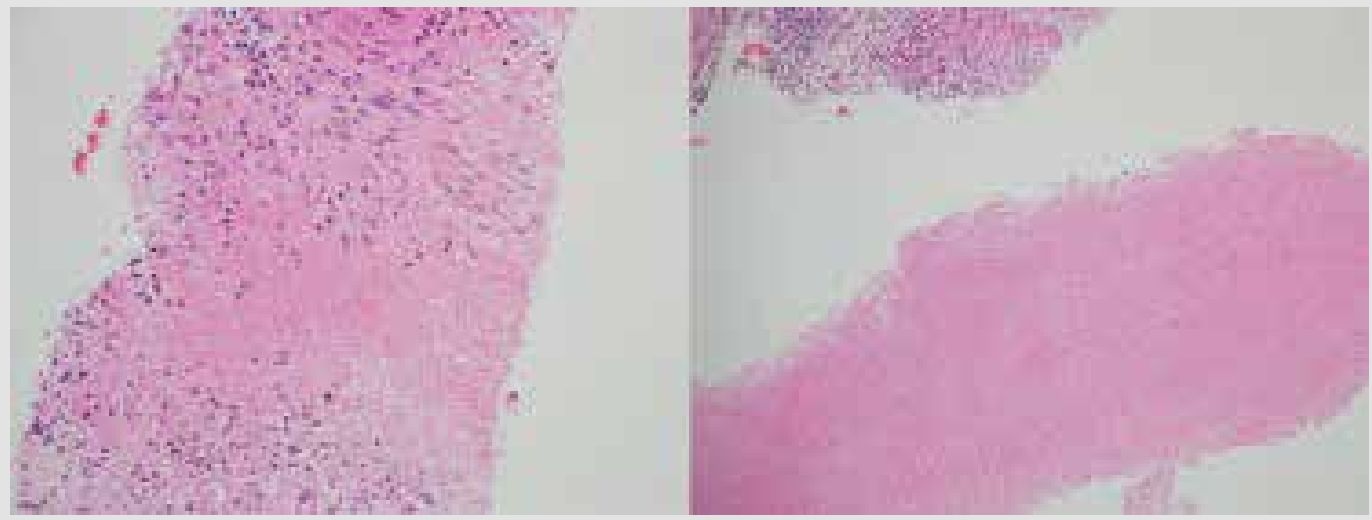

Figure 1. Histopathology of the LN biopsy showing central ncerotizing granulomatous areas, surrounded with giant cells

Prolactin was high at $1693 \mathrm{mIU} / \mathrm{I}$. HBV and HCV were negative. Our final diagnosis was KFD with primary Sjögren's syndrome.

\section{CONCLUSIONS}

Sjögren's syndrome can be associated with KFD and the finding of necrotizing granulomas in the lymph nodes should raise the possibility of such a combination. A high index of suspicion is required to identify the coexistence of Sjögren's syndrome with KFD.

\section{REFERENCES}

1. Zhang J, Yang J, Weng W-W, Zhu Y-J, Qiu H, Dong M-J. Kikuchi-Fujimoto disease associated with Sjogren's syndrome: a case report and review of the literature. Int J Clin Exp Med 2015;8:17061-17066.

2. Asano S, Akaike Y, Jinnouchi H, Muramatsu T, Wakasa H. Necrotizing lymphadenitis: a review of clinicopathological, immunohistochemical and ultrastructural studies. Hematol Oncol 1990;8:251-260.

3. Dorfman RF, Berry GJ. Kikuchi's histiocytic necrotizing lymphadenitis: an analysis of 108 cases with emphasis on differential diagnosis. Semin Diagn Pathol 1988:5:329-345.

4. Bosch X, Guilabert A, Miquel R, Campo E. Enigmatic Kikuchi-Fujimoto disease: a comprehensive review. Am J Clin Pathol 2004;122:141-152.

5. Miyashita Y, Yamaguchi M, Fujimoto W. Painful indurated erythema suggestive of Kikuchi-Fujimoto disease in a patient with primary Sjögren's syndrome. J Dermatol 2003;30:608-611. 